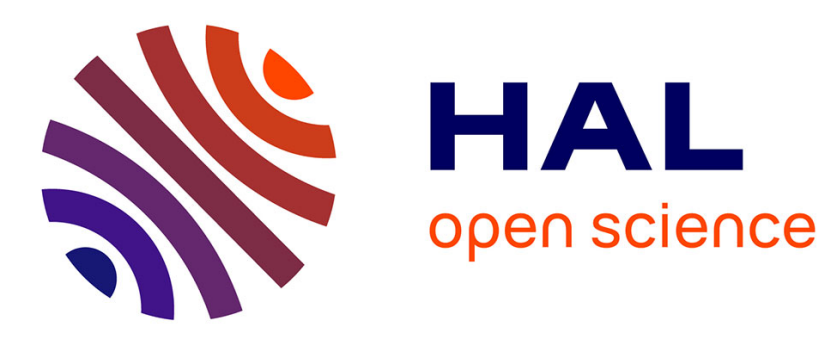

\title{
Torsional Stability of a U-Joint Based Parallel Wrist Mechanism Featuring Infinite Torsion \\ Guanglei Wu, Stéphane Caro
}

\section{To cite this version:}

Guanglei Wu, Stéphane Caro. Torsional Stability of a U-Joint Based Parallel Wrist Mechanism Featuring Infinite Torsion. In: Arakelian V., Wenger P. (eds) ROMANSY 22 - Robot Design, Dynamics and Control. CISM International Centre for Mechanical Sciences (Courses and Lectures), Vol. 584, Springer, Cham., pp.147-154, 2019, 10.1007/978-3-319-78963-7_20 . hal-02405586

\section{HAL Id: hal-02405586 \\ https://hal.science/hal-02405586}

Submitted on 11 Dec 2019

HAL is a multi-disciplinary open access archive for the deposit and dissemination of scientific research documents, whether they are published or not. The documents may come from teaching and research institutions in France or abroad, or from public or private research centers.
L'archive ouverte pluridisciplinaire HAL, est destinée au dépôt et à la diffusion de documents scientifiques de niveau recherche, publiés ou non, émanant des établissements d'enseignement et de recherche français ou étrangers, des laboratoires publics ou privés. 


\title{
Torsional Stability of a U-joint based Parallel Wrist Mechanism Featuring Infinite Torsion
}

\author{
Guanglei $\mathrm{Wu}^{1}$ and Stéphane Caro ${ }^{2}$ \\ 1 School of Mechanical Engineering, Dalian University of Technology, 116024 Dalian, China, \\ gwuedlut . edu.cn \\ 2 CNRS, Laboratoire des Sciences du Numérique de Nantes, UMR CNRS 6004, Ecole Centrale \\ de Nantes, 44321 Nantes, France, \\ stephane.carodls 2 n. fr
}

\begin{abstract}
In this paper, the dynamic stability problem of a parallel wrist mechanism is studied by means of monodromy matrix method. This manipulator adopts a universal joint as the ball-socket mechanism to support the mobile platform and to transmit the motion/torque between the input shaft and the end-effector. The linearized equations of motion of the mechanical system are established to analyze its stability according to the Floquet theory. The instable regions are presented graphically in various parametric charts.
\end{abstract}

Keywords: dynamic stability, parallel wrist mechanism, monodromy matrix, Floquet theory, torsional vibrations

\section{Introduction}

The parallel wrist mechanisms are intended for camera-orientating [9], minimally invasive surgical robots [12] and robotic joints [1], thanks to their large orientation workspace and high payload capacity. Besides, another potential application is that they can function as a tool head for complicated surface machining [19], where an unlimited torsional motion is desired to drive the cutting tools in some common material processing such as milling or drilling. For this purpose, a wrist mechanism [19] as shown in Fig. 1 was proposed with a number of advantages compared to its symmetrical counterparts, such as enhanced positioning accuracy [17], infinite rotation [1], structural compactness and low dynamic inertia [18]. The design of the manipulator is simplified by using a universal (U) joint supported by an input shaft to generate infinite input/output rotational motion. On the other hand, the $U$ joint suffers from one major problem, namely, it transforms a constant input speed to a periodically fluctuating one, which may induce vibrations and wear. This paper will investigate the dynamic stability problem, focusing on the aspect of the torsional stability.

To the best of the authors' knowledge, Porter [13] was the first to investigate this problem, where a single-degree-of-freedom linearized model was built to plot the stability chart by using the Floquet theory [8]. Later, similar modeling approaches were adopted to derive the nonlinear equations for the stability analysis of $U$ joint $[14,7$, $2,4,3]$. Moreover, multi-shaft system consisting of multiple shafts interconnected via 


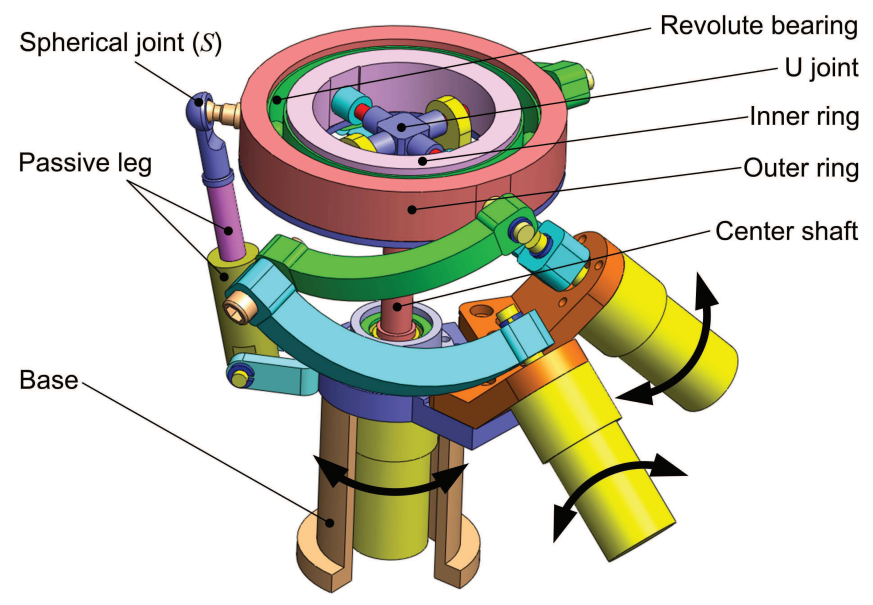

Fig. 1. CAD model of the parallel wrist mechanism.

Hooke's joints can also be handled using the previous various approaches [20,11]. Besides, lateral and coupled stability problem of the universal joint were studied $[10,15$, 6], too. According to the literature, the previous studies focus on single or multiple Ujoint mechanisms. On the other hand, a $U$ joint working as a transmitting mechanism in a parallel mechanism has not received the attention, which will be the subject in this work. From the reported works, common approaches to analyze the stability problem of the linear/nonlinear dynamic model of the system include Floquet theory, KrylovBogoliubov method, Poincaré-Lyapunov method, etc.. As the relationship between the input and output shaft rotating speeds of the $\mathrm{U}$ joint is periodic, the Floquet theory will be an effective approach to analyze the stability problem, which will be adopted in this work.

This paper investigates the dynamic stability analysis problem of the wrist mechanism by means of a monodromy matrix method. To this end, a linear model consisting of input and output shafts interconnected via a Hooke's joint is considered, and the linearized equations of motion of the system are obtained. Numerical study is carried out to assess the system stability and the effects of the parameters. Instable regions are identified from various parametric charts.

\section{Wrist Mechanism Architecture}

Figure 1 depicts the wrist mechanism, an asymmetrical spherical parallel manipulator (SPM). The mobile platform is composed of an outer and inner rings connected to each other with a revolute joint, the revolute joint being realized with a revolve bearing. The orientation of the outer ring is controlled by two limbs in-parallel, and it is constrained by a fully passive leg that is offset from the center of the mobile platform to eliminate the rotational motion around the vertical axis. Through a universal joint, the decoupled 
rotation of the inner ring is generated by the center shaft, which also supports the mobile platform to improve the positioning accuracy.

The architecture of the wrist mechanism is displayed in Fig. 2. Splitting the outer ring and the two parallel limbs as well as the passive one, the remaining parts of the manipualtor can be equivalent to a $\mathrm{U}$-joint mechanism. The center shaft is treated as the driving shaft and the inner ring is treated as a driven disk. The bend angle, i.e., the misalignment angle, is denoted by $\beta$, and the input/output angles are named as $\gamma_{1} / \gamma_{2}$, respectively.

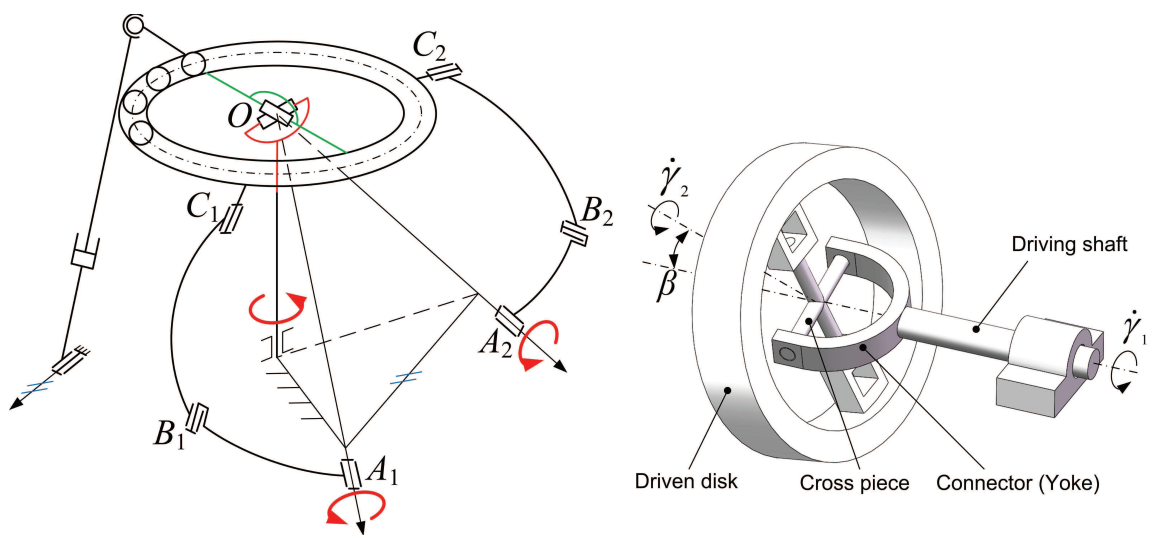

Fig. 2. Kinematic architectures of the wrist mechanism and its $U$ joint.

\section{Equation of Motion of Torsional Vibrations}

The equations of motion for the U-joint mechanism shown in Fig. 2 is deduced via a synthetical approach [3]. In accordance, the driving shaft and the driven disk are considered as two separate parts, as displayed in Fig. 3, where the cross piece connecting the input/output elements is considered as massless.

The equation of motion of torsional vibrations of the driving part can be written as

$$
J_{I} \ddot{\gamma}_{1}=-c_{1} \dot{\gamma}_{1}-k_{1} \gamma_{1}+M_{I}
$$

where $\gamma_{1}$ is the rotational coordinate of $J_{I}$, and $M_{I}$ is the reaction torque of the input part of the Hooke's joint. Moreover, $k_{1}$ and $c_{1}$ depict the torsional stiffness and viscous damper of the driving shaft, respectively.

On the other hand, the driven part is under the effect of the reaction torque $M_{O}$, for which the dynamic equation is written as

$$
c_{2} \dot{\gamma}_{2}+k_{2} \gamma_{2}=M_{O}=-J_{O}\left(\ddot{\gamma}_{2}+\dot{\omega}_{o}\right)
$$



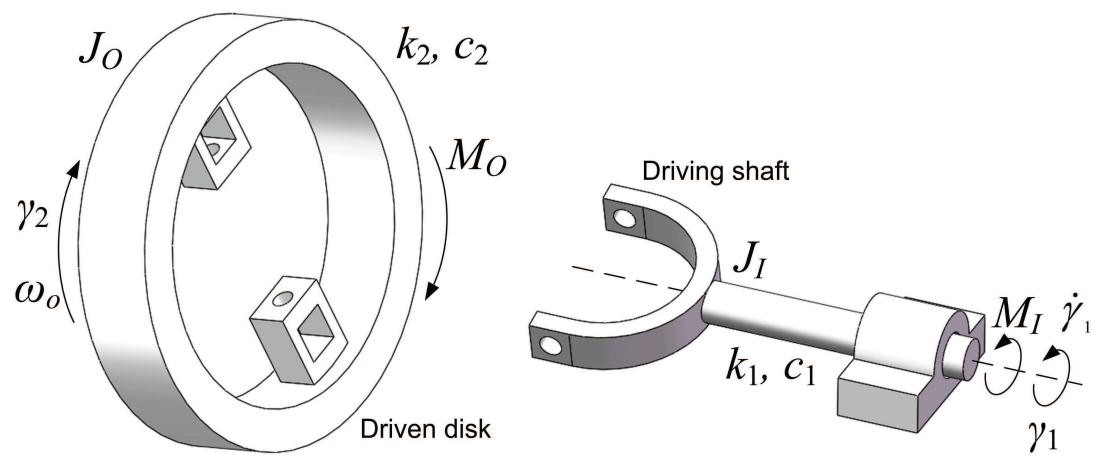

Fig. 3. The driving and driven parts of the U-joint mechanism.

where $\gamma_{2}$ is the rotational coordinate of $J_{O}$, and $k_{2}, c_{2}$ stand for the torsional stiffness and viscous damper of the driven shaft. Moreover, the relationship between the input torque and the output one of the Hooke's joint can be written as

$$
M_{O}=\frac{M_{I}}{\eta(t)}, \quad \eta(t)=\frac{\cos \beta}{1-\sin ^{2} \beta \sin ^{2}\left(\Omega_{0} t+\gamma_{1}\right)}
$$

where $\Omega_{0}$ denotes the constant velocity of the driving shaft, henceforth, the following equations of motion are derived

$$
\begin{aligned}
& J_{I} \ddot{\gamma}_{1}+c_{1} \dot{\gamma}_{1}+k_{1} \gamma_{1}-\eta(t) c_{2} \dot{\gamma}_{2}-\eta(t) k_{2} \gamma_{2}=0 \\
& J_{O}\left(\ddot{\gamma}_{2}+\dot{\omega}_{o}\right)+c_{2} \dot{\gamma}_{2}+k_{2} \gamma_{2}=0
\end{aligned}
$$

with

$$
\dot{\omega}_{o}=\eta(t) \ddot{\gamma}_{1}+\dot{\eta}(t)\left(\Omega_{0} t+\dot{\gamma}_{1}\right)
$$

Let $\tau$ be equal to $\Omega_{0} t+\gamma_{1}$. Some dimensionless parameters are defined as follows:

$$
\Omega=\frac{\Omega_{0}}{\sqrt{k_{1} / J_{I}}}, \zeta=\frac{c_{1}}{2 \sqrt{k_{1} J_{I}}}, \mu=\frac{c_{2}}{c_{1}}, v=\frac{J_{O}}{J_{I}}, \chi=\frac{k_{2}}{k_{1}}=\frac{1}{\eta(\tau)^{2}}
$$

Equations. (4) and (5) can be linearized and cast into a matrix form by discarding all the nonlinear terms, namely,

$$
\begin{aligned}
& {\left[\begin{array}{l}
\ddot{\gamma}_{1} \\
\ddot{\gamma}_{2}
\end{array}\right]+\left[\begin{array}{cc}
\frac{2 \zeta}{\Omega} & -\frac{2 \mu \zeta}{\Omega} \eta(\tau) \\
2 \eta^{\prime}(\tau)-\frac{2 \zeta}{\Omega} \eta(\tau) & \frac{2 \mu \zeta}{\Omega}\left(\frac{1}{v}+\eta^{2}(\tau)\right)
\end{array}\right]\left[\begin{array}{l}
\dot{\gamma}_{1} \\
\dot{\gamma}_{2}
\end{array}\right]} \\
& +\left[\begin{array}{cc}
\frac{1}{\Omega^{2}} & -\frac{\chi}{\Omega^{2}} \eta(\tau) \\
\eta^{\prime \prime}(\tau)-\frac{1}{\Omega^{2}} \eta(\tau) & \frac{\chi}{\Omega^{2}}\left(\frac{1}{v}+\eta^{2}(\tau)\right)
\end{array}\right]\left[\begin{array}{l}
\gamma_{1} \\
\gamma_{2}
\end{array}\right]=\left[\begin{array}{c}
0 \\
-\eta^{\prime}(\tau)
\end{array}\right]
\end{aligned}
$$

where primes denote differentiation with respect to $\tau$, thus, Eq. (8) consists of a set of linear differential equations with $\pi$-periodic coefficients. 


\section{Dynamic Stability Analysis}

The homogeneous parts of Eq. (8) should be considered sequentially to analyze the dynamic stability of the manipulator. Equation (8) can be expressed as:

$$
\ddot{\boldsymbol{\gamma}}+\mathbf{D} \dot{\boldsymbol{\gamma}}+\mathbf{E} \boldsymbol{\gamma}=\mathbf{0}
$$

with

$$
\begin{aligned}
& \ddot{\boldsymbol{\gamma}}=\left[\begin{array}{ll}
\ddot{\gamma}_{1} \ddot{\gamma}_{2}
\end{array}\right]^{T}, \dot{\boldsymbol{\gamma}}=\left[\begin{array}{ll}
\dot{\gamma}_{1} & \dot{\gamma}_{2}
\end{array}\right]^{T}, \boldsymbol{\gamma}=\left[\begin{array}{ll}
\gamma_{1} & \gamma_{2}
\end{array}\right]^{T} \\
& \mathbf{D}=\left[\begin{array}{cc}
\frac{2 \zeta}{\Omega} & -\frac{2 \mu \zeta}{\Omega} \eta(\tau) \\
2 \eta^{\prime}(\tau)-\frac{2 \zeta}{\Omega} \eta(\tau) & \frac{2 \mu \zeta}{\Omega}\left(\frac{1}{v}+\eta^{2}(\tau)\right)
\end{array}\right] \\
& \mathbf{E}=\left[\begin{array}{cc}
\frac{1}{\Omega^{2}} & -\frac{\chi}{\Omega^{2}} \eta(\tau) \\
\eta^{\prime \prime}(\tau)-\frac{1}{\Omega^{2}} \eta(\tau) \frac{\chi}{\Omega^{2}}\left(\frac{\Gamma^{2}}{v}+\eta^{2}(\tau)\right)
\end{array}\right]
\end{aligned}
$$

which can be represented by a state-space formulation, namely,

$$
\dot{\mathbf{x}}(t)=\mathbf{A}(t) \mathbf{x}(t)
$$

with

$$
\mathbf{x}(t)=\left[\begin{array}{l}
\boldsymbol{\gamma} \\
\dot{\gamma}
\end{array}\right], \mathbf{A}(t)=\left[\begin{array}{cc}
\mathbf{0}_{2} & \mathbf{I}_{2} \\
-\mathbf{E} & -\mathbf{D}
\end{array}\right]
$$

whence $\mathbf{A}(t)$ is a $4 \times 4 \pi$-periodic matrix. According to Floquet theory, the solution to equation system (11) can be expressed as

$$
\boldsymbol{\Phi}(\tau)=\mathbf{P}(\tau) e^{\tau \mathbf{R}}
$$

where $\mathbf{P}(\tau)$ is a $\pi$-periodic matrix and $\mathbf{R}$ is a constant matrix, which is related to another constant matrix $\mathbf{H}$, referred to as monodromy matrix, with $\mathbf{R}=\ln \mathbf{H} / \pi$. If the fundamental matrix is normalized so that $\mathbf{P}(0)=\mathbf{I}_{4}$, then $\mathbf{H}=\mathbf{P}(\pi)$.

The eigenvalues $\lambda_{i}, i=1,2,3,4$, of matrix $\mathbf{H}$, referred to as Floquet multipliers, govern the stability of the system. The system is asymptotically stable if and only if the real parts of all the eigenvalues $\lambda_{i}$ are non-positive [5]. Here, the matrix $\mathbf{H}$ is obtained numerically with the improved Runge Kutta Method [16] with a step size equal to $10^{-6}$, and and its eigenvalues are calculated to assess stability of the system. The monodromy matrix method is a simple and reliable method to determine the stability of parametrically excited systems.

\section{Numerical Study on Torsional Stability}

This section is devoted to numerical stability analysis, where the stability charts are constructed on the $\Omega_{0}-\beta$ and $k_{1}-\beta$ parametric planes to study the effect of parameters onto the system stability. From the CAD model of the robotic wrist, $\mu=1, v=10$, $J_{I}=0.001 \mathrm{~kg} \cdot \mathrm{m}^{2}, c_{1}=0.001 \mathrm{Nm} /(\mathrm{rad} / \mathrm{s})$.

Figure 4 depicts the stability chart $\Omega_{0}-\beta$ to detect the instability of the U-joint mechanism, with a constant stiffness $k_{1}=10 \mathrm{Nm} / \mathrm{rad}$, where the dotted zones represent 
the unstable parametric regions. When the rotating speed $\Omega_{0}$ of the driving shaft is lower than $11 \pi \mathrm{rad} / \mathrm{s}$, this system is always stable when the misalignment angle $\beta$ is between 0 and $30^{\circ}$. On the contrary, angle $\beta$ should be smaller than $5^{\circ}$ to guarantee dynamic stability of the parallel wrist mechanism when $\Omega_{0}$ is equal to $19 \pi \mathrm{rad} / \mathrm{s}$.

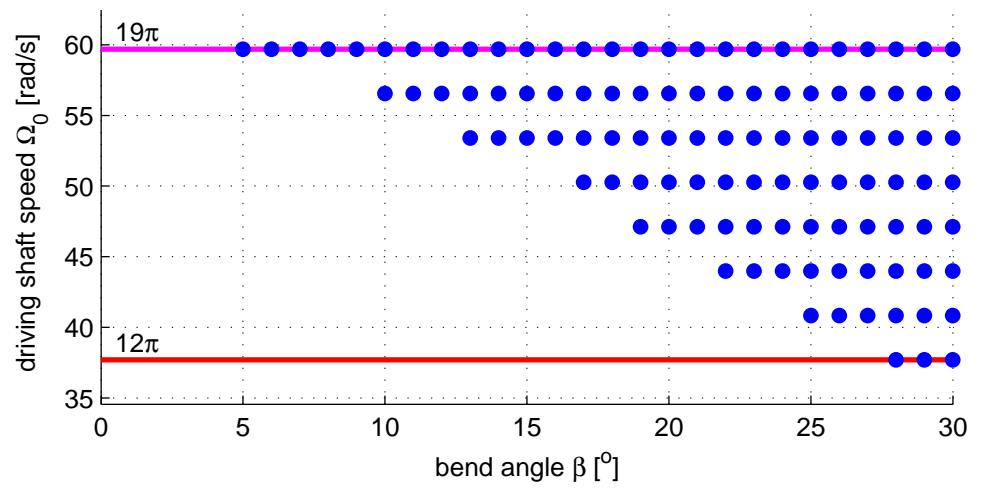

Fig. 4. Effects of the driving shaft speed $\Omega_{0}$ and bend angle $\beta$ onto the torsional stability of the parallel wrist with stiffness $k_{1}=10 \mathrm{Nm} / \mathrm{rad}$ (blue point means torsional dynamic instability).

Similarly, the influence of the torsional stiffness of the driving shaft and the misalignment angle to the stability is illustrated in Fig. 5, with the driving shaft speed $\Omega_{0}=19 \pi \mathrm{rad} / \mathrm{s}$. It is apparent that the higher the torsional stiffness of the input shaft, the more stable the parallel robotic wrist. The system is stable when $k_{1}>25 \mathrm{Nm} / \mathrm{rad}$.

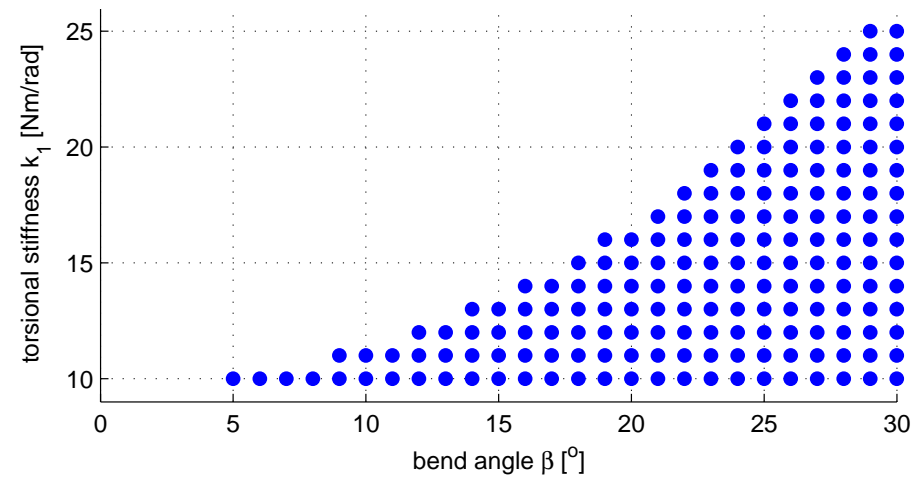

Fig. 5. Effects of the driving shaft stiffness $k_{1}$ and bend angle $\beta$ onto the dynamic torsional stability with speed $\Omega_{0}=19 \pi \mathrm{rad} / \mathrm{s}$. 


\section{Conclusion}

This paper dealt with the dynamic torsional stability analysis of a parallel wrist mechanism that contains a universal joint. Differing from the symmetrical counterparts, the asymmetrical architecture of this robotic wrist ensures an infinite torsional movement of the end-effector under a certain tilt angle. This unique feature allows the wrist mechanism to function as an active spherical joint or machine tool head, with a simple architecture.

The stability problem of the wrist mechanism due to the nonlinear input-output transmission of the universal joint is studied, where a linear model consisting of input and output shafts interconnected via a Hooke's joint is considered. The linearized equations of motion of the system are obtained, for which the stability problem is investigated by resorting to a monodromy matrix method. The approach used to analyze the torsional stability of the parallel robotic wrist is numerically illustrated, wherein the instable regions are presented graphically. Moreover, some critical parameters, such as torsional stiffness and rotating speeds, are identified. Future work includes the complete parametric stability analysis of the system as well as its lateral stability.

\section{Acknowledgement}

The reported work is supported by the Doctoral Scientific Research Foundation of Liaoning Province (No. 20170520134) and the Fundamental Research Funds for the Central Universities (No. DUT16RC(3)068).

\section{References}

1. Asada, H., Granito, J.: Kinematic and static characterization of wrist joints and their optimal design. In: IEEE Int. Conf. Robot. Autom., pp. 244-250 (1985)

2. Asokanthan, S.F., Hwang, M.C.: Torsional instabilities in a system incorporating a hooke's joint. J. Vib. Acoust. 118(3) (1996)

3. Bulut, G., Parlar, Z.: Dynamic stability of a shaft system connected through a hooke's joint. Mech. Mach. Theory 46(11), 1689-1695 (2011)

4. Chang, S.I.: Torsional instabilities and non-linear oscillation of a system incorporating a hooke's joint. J. Sound Vib. 229(4), 993-1002 (2000)

5. Chicone, C.: Ordinary Differential Equations with Applications. Springer, New York, NY (2006)

6. Desmidt, H.A., Wang, K.W., Smith, E.C.: Coupled torsion-lateral stability of a shaft-disk system driven through a universal joint. ASME J. Appl. Mech. 69(3), 261-273 (2002)

7. Éidinov, M.S., Nyrko, V.A., Éidinov, R.M., Gashukov, V.S.: Torsional vibrations of a system with hooke's joint. Soviet Applied Mechanics 12(3), 291-298 (1976)

8. Floquet, G.: Sur les équations différentielles linéaires à coefficients périodiques. Annales de l'École Normale Supérieure 12, 47-88 (1883)

9. Gosselin, C.M., Hamel, J.F.: The Agile Eye: a high-performance three-degree-of-freedom camera-orienting device. In: IEEE Inter. Conf. Robot. Autom., pp. 781-786 (1994)

10. H. Ota, M.K., Sugita., H.: Lateral vibrations of a rotating shaft driven by a universal joint: 1 st report, generation of even multiple vibrations by secondary moment. Bulletin of the JSME 27(231), 2002-2007 (1984) 
11. Kotera, T.: Instability of torsional vibrations of a system with a cardan joint. Memoirs of the Faculty of Engineering Kobe University 26, 19-30 (1980)

12. Li, T., Payandeh, S.: Design of spherical parallel mechanisms for application to laparoscopic surgery. Robotica 20(2), 133-138 (2002)

13. Porter, B.: A theoretical analysis of the torsional oscillation of a system incorporating a hooke's joint. ARCHIVE J. Mech. Eng. Sci. 3(4), 324-329 (1961)

14. Porter, B., Gregory, R.W.: Non-linear torsional oscillation of a system incorporating a hooke's joint. ARCHIVE J. Mech. Eng. Sci. 5(2), 191-209 (1963)

15. Saigo, M., Okada, Y., Ono, K.: Self-excited vibration caused by internal friction in universal joints and its stabilizing method. J. Vib. Acoust. 119(2), 221-229 (1997)

16. Szymkiewicz, R.: Numerical Solution of Ordinary Differential Equations. Academic Press (1971)

17. Wu, G., Bai, S., Kepler, J.: Mobile platform center shift in spherical parallel manipulators with flexible limbs. Mech. Mach. Theory 75, 12-26 (2014)

18. Wu, G., Caro, S., Bai, S., Kepler, J.: Dynamic modeling and design optimization of a 3-DOF spherical parallel manipulator. Robot. Auto. Syst. 62, 1377-1386 (2014)

19. Wu, G., Caro, S., Wang, J.: Design and transmission analysis of an asymmetrical spherical parallel manipulator. Mech. Mach. Theory 94, 119-131 (2015)

20. Zeman, V.: Dynamik der drehsysteme mit kardagelenken. Mech. Mach. Theory 13, 107-118 (1978) 